\title{
Sección de información
}

\section{Segundo Simposio Colombiano de Sicoprofilaxia Obstétrica}

Como informe a todos los obstetras del país especialmente a los interesados en sicoprofilaxis obstétrica (S.P.O.) he querido presentar un resumen sobre el desarrollo de este certamen científico.

Se efectuó en Medellín en los días 4 a 7 del pasado mes de mayo con asistencia de 76 médicos, la mayoría de los cuales provenía de otras ciudades del país: Barranquilla, Cartagena, Cúcuta, Bucaramanga, Pamplona, Cali, Armenia, Pereira, Manizales y Bogctá.

Esta reunión obedeció al plan propuesto en el Primer Simposio, organizado por el EMESFAO (Equipo de Analgesia Obstétrica de Bogotá) en 1959 sobre difusión y perfeccionamiento de la S.P.O. en Colombia. La organización estuvo a cargo del Instituto de S.P.O. de Medellín, del Equipo de la Clínica Luz Castro de G. y del Equipo del Hospital de San Vicente de Paúl, contando con el apoyo de la Facultad de Medicina de la U., de A. de la Secretaría Departamental de Higiene, de la Secretaría Municipal de Higiene y de la Clínica Fabricato,

La colaboración científica estuvo a cargo, a más de los equipos locales, de la SCOSS (Sociedad Colombiana de Obstetricia Sicosomática) con participación de representantes de todas sus sedes (Bogotá, Barranquilla, Cúcuta, Bucaramanga, Pereira y Cali) y de la Sociedad de S.P.O.

Ponencias. E1 tema sobre "Medicación en pacientes preparadas" fue desarrollado por la Sociedad Col. de S.O. Se hizo una amplia revisión farmacológica y farmacodinámica de las diferentes drogas usadas durante el trabajo, de sus indicaciones en pacientes preparadas y su acción sobre la contractilidad uterina y especialmenté sobre la corteza cerebral. 
El comentario de esta ponencia estuvo a cargo de la SCOSS. Hicimos resaltar la importancia y el valor de esta contribución pero hicimos notar también la falta de experiencia y conceptos personales. En Co.ombia ya tenemos buena práctica en S.P.O., superior a la de otros países latincamericanos, por tanto no debemos ya limitarnos exclusivamente a citar autores extranjeros, sino que debemos y podemos deducir hechos concretos de nuestra practica diaria.

\section{Intervenciones Obstétricas en Pacientes Preparadas}

Esta discutida ponencia fue desarrollada por el Instituto F. Lamaze de Cali. Los ponentes, mencionando de paso la aplicación profiláctica de forceps, explicaron con lujo de detalles (transparencias, película, cinta magnetofónica) su técnica de revisión del cuello y de la cavidad uterina, ésta última practicada por ellos en forma sistemá tica después de todo parto. Surgen muchas dudas respecto de la posibilidad de estas maniobras. Fué una lástima que en el Simposio no se hubiera dispuesto de tiempo suficiente para discusiones en mesas redondas, donde se hubieran podido precisar muchos conceptos no solo sobre éste tema sino sobre otros muchos. En todo caso reconocemos originalidad en la técnica y en los conceptos de los ponentes; ellos han abierto un nuevo campo de investigación y estudio. Esto constituye de por sí un avance de la S.P. nacional y un indiscutible mérito de ellos.

\section{Unificación de Historia Clinica y Valoración de Resultades}

La primera parte de este tema fue desarrollada por la SCOSS, sede de Bocotá y la sequnda, la referente a valoración de resultados, por la SCOSS, sede de Barranquilla.

El modelo de historia que presentamos es el que la SCOSS viene empleando desde el año pasado. Es un esqueleto sencillo, práct:co y de fácil tabulación. Le fueron propuestas algunas modificacionrs que nosotros consideramos sin mayor importancia. Por tanto la SCOSS seguirá usando su historia clínica. Nuestro propósito no era imponer determinado tipo de historia, sino llevar el ánimo de los asistentes al Simposio, a la necesidad de hacer historias clínicas.

En cuanto a la valoración de resultados se hizo notar también la falta de tiempo suficiente, no sólo para la comisión de conclusiones, sino para una mesa redonda cspecial, de donde se hubiesen obtenido resultados más prácticos que hubiesen dejado satisfechos a todos los equipos asistentes. Lo cual no fue así. 
El Dr. Navas Uribe, de la SCOSS de Barranquilla, propuso $-\mathrm{n}-$ te todo simplificar la clasificación de resultados aprobada en el Primer Simposio (casos excelentes, buenos, regulares y malos), reduciéndolos a tres grupos: $1^{\circ}$ éxitos totales, que comprende los excelentes de la clasificación anterior; $2^{\circ}$ éxitos parciales, o sea los buenos y regulares y $3^{\circ}$ fracasos, o sea los casos malos de la clasificación anterior.

Propuso también cambiar la denominación, muy atinada y honradamente, de este sistema que hemos venido tratando de difundir en todo el país, para estar más de acuerdo con la realidad de los hechos y de los resultados. El término "Método sicoprofiláctico de loz dolores del parto" no es acertado, pues la supresión de los dolores solo se consigue $€ n$ un porcentaje ínfimo. La denominación actual más accptada "Preparación Sicoprofiláctica de las embarazadas para el parto", también es susceptible de modificación por ser muy vaga e imprecisa, en nuestro sentir.

El término propuesto por el Dr. Navas de "Parto tranquilo" no fue aceptado por los comentaristas (Sociedad Col. de S. P.) aduciendo razones poco valederas y sin presentar tampcco un término sustitutivo. Personalmente no nos satisface plenamente el término del $D_{r}$. Navas pero sí cstamos de acuerdo en que la actual denominación del sistema dibe modificarse totalmente para estar más de acuer do con los resultados. Para tratar este punto la SCOSS ya ha nombrado una comisión que presentará un detenido estudio en el próximo Simposio.

Temas Libres.- Todas las sedes de la SCOSS (Cúcuta, Barranquilla, Bucaramanga, Cali y Bogotá) presentaron un informe cstadístico de sus labores hasta el presente. Merece especial mención la labor del Dr. Isaías Arenas Buenahora en Bucaramanga, por la presentación de su magnífica película sonora y en colore $e_{S}$ elaborada técnicamente con sus propios medios y con la colaborarión de sus compañeros de equipo y de sus pacientes particulares. Estz esfuerzo fue reconocido y aplaudido por los asistentes al Simposio.

La Clínica D. Restrepo presentó una estadística nutrida, si se ticne en cuenta el poco tiempo de trabajo: algo más de 600 casos. Nos llamó la atención el número elevado de fracasos: $22 \%$. Es cierto que hay un criterio muy severo en la calificación de los casos pero deben existir causas concretas de fracasos que no fueron analizadas por los autores. La clinica Luz Castro de Gutiérrez presentó sus primeras experiencias cen el método: sobre 315 pacientes preparadas presentó resultados de $17 \%$ casos bien controlados, con un $34 \%$ de casos excelentes y tan sólo un $10 \%$ de fracasos. Por las condiciones de comodidad y magnifico cquipo consideramos que esta clínica llegará a ser cn breve ticmpo el priner centro de S.P.O. del país. 
El EMESFAO presentó los siguientes trabajos: "Forma de trabajo actual del Equipo" en la que hicimos resaltar la importancia de dictar a las pacientes al comienzo del embarazo un corto curso sobre higiene y cuidados prenatales, aspectos emocionales y conferencia para maridos. "Datos estadísticos generales del EMESFAO" y "Causas de fracasos en S.P.O." trabajos que próximamente iran a ser publicados en la revista.

Otros equipos de Medellin presentaron también interesantes trabajos, El Instituto de S.P.O. de Medellín presentó un correlato sobre preparación extemporánea además de una exposición sobre su forma de trabajo y datos estadísticos. El Dr. Fabio Alvarez presentó un informe sobre el desarrollo del método en los centros materno infantiles de Medellin.

La Sociedad Col. de S.P.O. presentó también un trabajo sobre preparación extemporánea que provocó una interesante discusión sobre si dicho sistema era o no S. P. O. biano.

Conclusiones: Intensificar la P.S.O. en todo el territorio Colom-

Próxima Sede: Se acordó reunir el III Simposio el año entran te (1961) en la ciudad de Bucaramanga, en la primera semana de mayo.

Ponencias para el III Simposio: Con el fin de dar un aspecto experimental al método fueron acordados los siguientes temas:

1. Reacciones sicomotoras en S.P.O. Ponente: SCOSS sede de Barranquilla.

$2^{\circ}$ Estudio experimental del testimonio materno en S.P.O. (espectrosicómetro) Dr. Gustavo Isaza Mejia.

3: Otras técnicas de condicionamiento. Clínica D. Restrepo.

En resumen, el II Simposio constituyó otro éxito y una demostración más de que el sistema ha venido difundiéndose progresivamente en el país. La organización fue muy buena y los asistentes fuimos objeto de numerosas atenciones. Esta reunión como la próxima en Bucaramanga, pondrá de presente el gran interés de los obstetras colombianos para la elaboración de la ponencia sobre P.S.P. para el Congreso latinoamericano de Obstetricia y Ginecología que se reunirá en Bogotá en el año de 1962.

Carlos R. Silva Mojica 